\title{
Quantum Mathematical Integrated Information Theory: From the Classical Version
}

\author{
Siddharth Sharma \\ E-mail : wise.plant.sid@gmail.com
}

\begin{abstract}
This paper is an attempt to give a Quantum theory of Mathematical integrated information theory which is mathematical version of integrated information theory by Masafumi Oizumi, Larissa Albantakis, Giulio Tononi [2]. Using the definitions of Classical Mathematical Integrated Information Theory [1]. And considering that the Quantum theory is given by the functor which maps from a category whose objects is topology to a linear category whose objects are Hilbert spaces indexed with the objects from previous category. Also, we will be using the definition of conditional density matrix to define repertoire. We will be also discussing the relationship between consciousness and entanglement
\end{abstract}

\section{Introduction}

In the following sections we will develop a theory of consciousness of a quantum system, consider definitions from Classical Mathematical Integrated Information Theory [1] and try to Quantise it by considering a functor, $\mathcal{F}$ which maps from classical system to the quantum system. And there by using classical definition and generating their quantum enalog.

The parts in italic are from Classical Mathematical Integrated Information Theory and parts in normal font is the explanation for Quantum Mathematical Integrated Information Theory

\section{Postulates}

“...... if we consider a set of elements $\Lambda$ then system is the topology, $\mathcal{T}$ of the set $\Lambda$ and mechanisms, $M$ is open subset of set $\Lambda$ i.e. $M$ belongs $\mathcal{T} ; M \in \mathcal{T}$......."

A set of elements $\Lambda$ then system is the topology, $\mathcal{T}$ of the set $\Lambda$ and mechanisms, $M$ is open subset of set $\Lambda$ i.e. $M$ belongs $\mathcal{T} ; M \in \mathcal{T}$. Let $\mathcal{F}$ be a functor from topological category $\mathcal{L}$ to linear category $\mathcal{H}$. With $\operatorname{Obj}(\mathcal{L}):=\mathcal{T}$ and $\operatorname{Obj}(\mathcal{H}):=\left\{\otimes_{\alpha \in M} \mathbb{H}_{\alpha}\right\}_{M \in \mathcal{T}}$. Considering state as endomorphism if $s$ is the state of mechanism $M, \rho_{M}:=\mathcal{F}(s)$ is endomorphism of $\mathbb{H}_{M}$, where $\mathbb{H}_{M}:=\otimes_{\alpha \in M} \mathbb{H}_{\alpha} \cdot \rho_{M}$ can be written as $\frac{1}{N} \sum_{\alpha} \otimes_{\alpha_{i} \in \Lambda} \sigma(M)_{\alpha_{i}}$ where $\sigma(M)_{\alpha}:=$ $\left\{\begin{array}{l}\rho_{\alpha_{i}} \text { if } \alpha_{i} \in M \\ \frac{\text { id } d_{\alpha_{i}}}{\operatorname{dim} \mathbb{H}_{\alpha_{i}}} \text { if } \alpha_{i} \notin M\end{array}\right.$ and $\alpha:=\left(\alpha_{i}\right)_{i}$. For some $\rho_{\alpha} \in E n d \mathbb{H}_{\alpha}$ with trace equals to one and are Hermitian. Here $N$ is such that trace of $\rho_{M}$ is equal to 1 . 


\section{Mechanisms}

- "INFORMATION: , a mechanism in a state generates information only if it constrains the states of a system that can be its possible causes and effects - its causeeffect repertoire. The more selective the possible causes and effects, the higher the cause-effect information (cei) specified by the mechanism"

- Definition: Cause-effect repertoire: "It ............is set of all the probability distributions of states of mechanisms called Perviews, $P$ knowing that mechanism, $M$ is in state, $s$ mathematically speaking $\left\{p^{x}\left(P \mid M_{S}\right)\right\}_{\substack{P \in T \\ P \neq M}}$ where $P \in \mathcal{T}$ and $P \neq M, p$ represents probability distribution function, $x \in\{e, c\}$ where e represents effect and c represents cause."

Here $p^{x}\left(P \mid M_{S}\right)$ is conditional probability density so the quantum enalog of it is conditional density matrix,

$$
\varrho^{x}\left(\rho_{P} \mid \rho_{M}\left(t_{0}\right)\right):=\frac{\operatorname{tr}_{M}\left(\rho_{P}(t) \rho_{M}\left(t_{0}\right)\right)}{\operatorname{tr}\left(\rho_{P}(t) \rho_{M}\left(t_{0}\right)\right)}
$$

Where,

$$
\operatorname{tr}_{M}: \text { End }\left(\mathbb{H}_{\Lambda}\right) \rightarrow \text { End }\left(\mathbb{H}_{\Lambda}\right)
$$

such that $\operatorname{tr}_{M}$ of $\rho_{N}$ is $\frac{1}{N} \sum_{\alpha} \otimes_{\alpha_{i} \in \Lambda} \psi(M, N)_{\alpha_{i}}$ for some $N \in \mathcal{T}$. Here

$$
\psi(M, N)_{\alpha_{i}}:=\left\{\begin{array}{c}
\sigma(N)_{\alpha_{i}} \text { if } \alpha_{i} \notin M \\
\operatorname{tr}_{\alpha_{i}}\left(\sigma(N)_{\alpha_{i}}\right) \text { id } \alpha_{\alpha_{i}} \text { if } \alpha_{i} \in M
\end{array}\right.
$$

And tr: End $\left(\mathbb{H}_{\Lambda}\right) \rightarrow \mathbb{C}$, is trace in denominator is over $\Lambda$. Also $x:=e$ for $t>t_{0}$ and $x:=c$ for $t<t_{0}$. Note that $\operatorname{tr}_{\alpha_{i}}$ : End $\left(\mathbb{H}_{\alpha_{i}}\right) \rightarrow \mathbb{C}$, is the trace over $\alpha_{i} . \alpha:=$ $\left(\alpha_{i}\right)_{i}$

In the rest of the sections and subsections we need to replace, $\varrho^{x}\left(\rho_{P} \mid \rho_{M}\left(t_{0}\right)\right)$ with $p^{x}\left(P \mid M_{S}\right)$ and the term generated by $\varrho^{x}\left(\rho_{P} \mid \rho_{M}\left(t_{0}\right)\right)$ with term generated by $p^{x}\left(P \mid M_{S}\right)$ to develop the quantum enalog for the classical one.

- Definition: Cause information (ci) and effect information (ei): Let $\mathcal{D}$ be a distance function over probability distribution space. Then the information is defined as follows

$$
x i\left(P \mid M_{s}\right):=\mathcal{D}\left(p^{x}\left(P \mid M_{S}\right), p^{x}(P \mid \varnothing)\right)
$$

Where unconstrained cause repertoire (same on the effect side), $p^{x}\left(P \mid M_{S}\right)$ and $x \in\{e, c\}$.

It is straight forward to see that the enalog to $\boldsymbol{c i}$ and $\boldsymbol{e i}$ is gained by replacing

- $p^{x}\left(P \mid M_{S}\right)$ with $\varrho^{x}\left(\rho_{P} \mid \rho_{M}\left(t_{0}\right)\right)$ 
- $p^{x}(P \mid \varnothing)$ with $\varrho^{x}\left(\rho_{P} \mid \rho_{\varnothing}\left(t_{0}\right)\right)$

- $\mathcal{D}$ with $\mathcal{D}_{Q}$

- $x i$ with $Q x i$

$$
\operatorname{Qxi}\left(\rho_{P} \mid \rho_{M}\left(t_{0}\right)\right):=\mathcal{D}_{\mathcal{Q}}\left(\varrho^{x}\left(\rho_{P} \mid \rho_{M}\left(t_{0}\right)\right), \varrho^{x}\left(\rho_{P} \mid \rho_{\emptyset}\left(t_{0}\right)\right)\right)
$$

Where $\mathcal{D}_{\mathcal{Q}}$ is quantum distance function unconstrained cause repertoire (same on the effect side), $\varrho^{x}\left(\rho_{P} \mid \rho_{\varnothing}\right)$ and $x \in\{e, c\}$.

Definition: Cause-effect information (cei): Cause-effect information of a mechanism, $M$ in state, $s$ is given by:

$$
\operatorname{cei}\left(P \mid M_{s}\right):=\min \left\{e i\left(P \mid M_{s}\right), c i\left(P \mid M_{s}\right)\right\}
$$

Quantum Cause-effect information of a mechanism can be given by:

$$
Q c e i\left(\rho_{P} \mid \rho_{M}\left(t_{0}\right)\right):=\min \left\{e i\left(\rho_{P} \mid \rho_{M}\left(t_{0}\right)\right), c i\left(\rho_{P} \mid \rho_{M}\left(t_{0}\right)\right)\right\}
$$

- "INTEGRATION: Let $p^{x}\left(P \mid \widehat{M}_{s}, \breve{M}_{s}\right)$ be probability distribution of states of states of system $\mathcal{T}$ knowing that mechanism $\widehat{M}$ and $\breve{M}$ are in state s. Where $\widehat{M} \cup \breve{M}:=M, \widehat{M}, \breve{M} \neq$ $M$ and $\widehat{M}, \breve{M} \in \mathcal{T}$ then,

$$
\varphi^{x}\left(P \mid M_{s}\right):=\min \left\{\mathcal{D}\left(p^{x}\left(P \mid M_{s}\right), p^{x}\left(P \mid \widehat{M}_{s}, \breve{M}_{s}\right)\right)\right\}_{\widehat{M} \cup \breve{M}:=M}
$$

Where $x \in\{e, c\}$ and integrated cause-effect information of mechanism, $M$ and in state, $s$ is:

$$
\varphi\left(P \mid M_{s}\right):=\min \left\{\varphi^{e}\left(P \mid M_{s}\right), \varphi^{c}\left(P \mid M_{s}\right)\right\} "
$$

The quantum enalog to $\varphi^{x}\left(P \mid M_{S}\right)$ can be given as.

$$
\varphi^{x}\left(\rho_{P} \mid \rho_{M}\left(t_{0}\right)\right):=\min \left\{\mathcal{D}\left(\varrho^{x}\left(\rho_{P} \mid \rho_{M}\left(t_{0}\right)\right), \varrho^{x}\left(\varrho^{x}\left(\rho_{P} \mid \rho_{\widetilde{M}}\left(t_{0}\right)\right) \mid \rho_{\widetilde{M}}\left(t_{0}\right)\right)\right)\right\}_{\overparen{M} \cup \widetilde{M}:=M}
$$

Where minimum is taken over $\widehat{M} \cup \breve{M}:=M, \widehat{M}, \breve{M} \neq M$ and $\widehat{M}, \breve{M} \in \mathcal{T}$ then, and $x \in$ $\{e, c\}$.

The quantum enalog to $\varphi\left(\rho_{P} \mid \rho_{M}\left(t_{0}\right)\right)$ can be given as

$$
\varphi\left(P \mid M_{S}\right):=\min \left\{\varphi^{e}\left(\rho_{P} \mid \rho_{M}\left(t_{0}\right)\right), \varphi^{c}\left(\rho_{P} \mid \rho_{M}\left(t_{0}\right)\right)\right\}
$$

- "EXCLUSION: It is plain and simple to see MICE

$$
\varphi^{\max }\left(M_{S}\right):=\max \left\{\varphi\left(P \mid M_{S}\right)\right\}_{\substack{P \in T \\ P \neq M}}
$$

That is maximum of all the integrated cause-effect information taken over all the preview not equals to mechanism, $M$. If $\varphi^{\max }\left(M_{s}\right)>0$ then it constitutes a concept, which is defined as follows 


$$
\mathcal{C}\left(M_{S}\right):=\left(\varphi^{\max }\left(M_{s}\right), p^{c}\left(M_{s}\right), p^{e}\left(M_{s}\right)\right) "
$$

The quantum enalog to $\varphi^{\max }\left(M_{S}\right)$ can be given by replacing $\varphi\left(P \mid M_{S}\right)$ with $\varphi\left(\rho_{P} \mid \rho_{M}\left(t_{0}\right)\right)$

$$
\varphi^{\max }\left(\rho_{M}\left(t_{0}\right)\right):=\max \left\{\varphi\left(\rho_{P} \mid \rho_{M}\left(t_{0}\right)\right)\right\}_{\substack{P \in T \\ P \neq M}}
$$

The quantum enalog to $\mathcal{C}\left(M_{S}\right)$ can be given by

$$
\mathcal{Q C}\left(\rho_{M}\left(t_{0}\right)\right):=\left(\varphi^{\max }\left(\rho_{M}\left(t_{0}\right)\right), \rho_{M}{ }^{c}\left(t_{0}\right), \rho_{M}{ }^{e}\left(t_{0}\right)\right)
$$

\section{Systems of mechanisms}

"INFORMATION: Conceptual structure, $\mathcal{C} \mathcal{S}(\mathcal{U})$ where $\mathcal{U} \subset \mathcal{T}$, is subsystem.

$$
\mathcal{C S}(\mathcal{U}):=\left(\mathcal{C}\left(M_{S}\right)\right)_{\substack{M \in U \\ s \in S}}
$$

Where $\mathbb{S}$ is set of all possible state. The conceptual information, $\mathcal{C J}$ of conceptual structure, $\mathcal{C S}(\mathcal{U})$ is linear functional on space of conceptual structure space and is defined as:

$$
\mathcal{C J}(\mathcal{C S}(\mathcal{U})):=\sum_{\substack{M \in U \\ s \in S}} \varphi^{\max }\left(M_{S}\right) \mathcal{J}(\mathcal{U})
$$

Where $\mathcal{J}(\mathcal{U}):=\max \left\{\operatorname{cei}\left(P \mid M_{S}\right)\right\}_{\substack{P \in T \\ M \in U}}$. This mathematical expression suites the definition as, it is increases with increase in number of points and also with $\varphi^{\text {max }}$."

The quantum enalog to $\mathcal{C} \mathcal{S}(\mathcal{U})$ can be given as, is given by replacing $\mathcal{C}\left(M_{S}\right)$ with $\mathcal{Q C}\left(\rho_{M}\left(t_{0}\right)\right)$

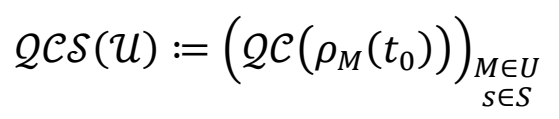

The quantum enalog to $\mathcal{C J}(\mathcal{C S}(\mathcal{U}))$ can be given as, is given by replacing $\varphi^{\max }\left(M_{S}\right)$ with $\varphi^{\max }\left(\rho_{M}\left(t_{0}\right)\right)$ and replacing $\mathcal{J}(\mathcal{U})$ with $\mathcal{Q} \mathcal{J}(\mathcal{U})$.

$$
\mathcal{Q C J}(\mathcal{L C S}(\mathcal{U})):=\sum_{\substack{M \in U \\ t_{0} \in R}} \varphi^{\max }\left(\rho_{M}\left(t_{0}\right)\right) \mathcal{Q J}(\mathcal{U})
$$

where $\mathcal{Q J}(\mathcal{U}):=\max \left\{Q \operatorname{cei}\left(\rho_{P} \mid \rho_{M}\left(t_{0}\right)\right)\right\}_{\substack{P \in T \\ M \in U}}$

- INTEGRATION: A set of elements can be conscious only if its mechanisms specify a conceptual structure that is irreducible to non-interdependent components (strong integration). Strong integration/irreducibility $\Phi$ is assessed by partitioning the set of elements into subsets with unidirectional cuts. 


$$
\Phi(\mathcal{U}):=\min \{\Delta(\mathcal{C S}(\mathcal{U}), \mathcal{C} \mathcal{S}(\widehat{\mathcal{u}})+\mathcal{C} \mathcal{S}(\breve{u}))\}_{\substack{\hat{u} \cup \check{u}=U \\ \hat{u}, \breve{u} \neq U \\ \widehat{u}, \breve{u} \subset T}}
$$

Where minimum is taken over all $\widehat{U}, \breve{u}$, satisfying three conditions:

- $\hat{U}, \breve{u} \subset T:$ i.e., $\widehat{u}, \breve{u}$ belongs to the system

- $\hat{\mathcal{U}} \cup \breve{u}=U$ : i.e., union of $\widehat{\mathcal{U}}, \breve{u}$ is $\mathcal{U}$

- $\widehat{u}, \breve{u} \neq U$ : i.e. None of $\widehat{u}, \breve{u}$ equals to $\mathcal{U}$

where $\Delta$ is distance function over space of conceptional structure and could be defined as follows:

$$
\Delta(\mathcal{C S}(\mathcal{U}), \mathcal{C S}(\mathcal{V})):=|\mathcal{C J}(\mathcal{C S}(\mathcal{U}))-\mathcal{C J}(\mathcal{C S}(\mathcal{V}))|
$$

Where $\mathcal{U}, \mathcal{v} \in \mathcal{T}$.

The quantum enalog to $\Phi(\mathcal{U})$ can be given as, is given by replacing $\mathcal{C} \mathcal{S}$ with $\mathcal{Q} \mathcal{C} \mathcal{S}$

$$
\Phi_{\mathcal{Q}}(\mathcal{U}):=\min \{\Delta(\mathcal{L C S}(\mathcal{U}), \mathcal{Q} \mathcal{C} \mathcal{S}(\widehat{\mathcal{u}})+\mathcal{Q} \mathcal{C} \mathcal{S}(\breve{u}))\}_{\substack{\hat{u} \cup \check{u}=U \\ \widehat{u}, \breve{u} \neq U \\ \widehat{u}, \breve{u} \subset T}}
$$

The quantum enalog to $\Delta(\mathcal{C S}(\mathcal{U}), \mathcal{C S}(\mathcal{V}))$ can be given as, is given by $\mathcal{C} \mathcal{S}$ with $Q \mathcal{C} \mathcal{S}$ and $\mathcal{C J}$ with $\mathcal{Q} \mathcal{C J}$.

$$
\Delta(\mathcal{Q} \mathcal{S}(\mathcal{U}), \mathcal{Q} \mathcal{S}(\mathcal{V})):=|\mathcal{Q} \mathcal{J}(\mathcal{C S}(\mathcal{U}))-\mathcal{Q} \mathcal{J J}(\mathcal{C S}(\mathcal{V}))|
$$

- EXCLUSION: Of all overlapping sets of elements, only one set can be conscious - the one whose mechanisms specify a conceptual structure that is maximally irreducible (MICS) to independent components. A local maximum of integrated information $\Phi^{\text {max }}$ (over elements, space, and time) is called a complex.

It is synonymous to MICE.

$$
\Phi^{\max }(\mathcal{T}):=\max \{\Phi(\mathcal{U})\}_{\mathcal{U} \mathcal{T}}
$$

The maximum is taken over all the subsets, $\mathcal{U}$ of the system, $\mathcal{T}$. Where $\Phi^{\max }(\mathcal{T})$ is measure of consciousness of system $\mathcal{T}$.

The quantum enalog to $\Phi^{\max }(\mathcal{T})$ can be given as, is given by

$$
\Phi_{Q}^{\max }(\mathcal{T}):=\max \left\{\Phi_{\mathcal{Q}}(\mathcal{U})\right\}_{\mathcal{U \subset \mathcal { T }}}
$$

The maximum is taken over all the subsets, $\mathcal{U}$ of the system, $\mathcal{T}$. Where $\Phi_{Q}^{\max }(\mathcal{T})$ is measure of consciousness of quantum system $\mathcal{T}$. 


\section{Entanglement and Consciousness}

Let $\left\{\mathbb{H}_{M}\right\}_{M \in \mathcal{T}}$ be a Quantum system, then the set of repertoires is:

$$
\left\{\left\{\varrho^{x}\left(\rho_{P} \mid \rho_{M}\left(t_{0}\right)\right)\right\}_{\substack{P \in T \\ P \neq M}}\right\}_{M \in \mathcal{T}}
$$

Where repertoire is:

$$
\varrho^{x}\left(\rho_{P} \mid \rho_{M}\left(t_{0}\right)\right):=\frac{\operatorname{tr}_{M}\left(\rho_{P}(t) \rho_{M}\left(t_{0}\right)\right)}{\operatorname{tr}\left(\rho_{P}(t) \rho_{M}\left(t_{0}\right)\right)}
$$

We know that QMICE, $\varphi^{\max }\left(\rho_{M}\left(t_{0}\right)\right)$ is $\min \left\{\varphi^{e}\left(\rho_{P} \mid \rho_{M}\left(t_{0}\right)\right), \varphi^{c}\left(\rho_{P} \mid \rho_{M}\left(t_{0}\right)\right)\right\}$, where

$$
\varphi^{x}\left(\rho_{P} \mid \rho_{M}\left(t_{0}\right)\right):=\min \left\{\mathcal{D}\left(\varrho^{x}\left(\rho_{P} \mid \rho_{M}\left(t_{0}\right)\right), \varrho^{x}\left(\varrho^{x}\left(\rho_{P} \mid \rho_{\widehat{M}}\left(t_{0}\right)\right) \mid \rho_{\breve{M}}\left(t_{0}\right)\right)\right)\right\}_{\substack{\widehat{M} \cup \breve{M}:=M \\, \widehat{M}, \breve{M} \neq M \\ \widehat{M}, \breve{M} \in T}}
$$

And

$$
\varrho^{x}\left(\varrho^{x}\left(\rho_{P} \mid \rho_{\widehat{M}}\left(t_{0}\right)\right) \mid \rho_{\breve{M}}\left(t_{0}\right)\right):=\frac{\operatorname{tr}_{\breve{M}}\left(\operatorname{tr}_{\widehat{M}}\left(\rho_{P}(t) \rho_{\widehat{M}}\left(t_{0}\right)\right) \rho_{\breve{M}}\left(t_{0}\right)\right)}{\operatorname{tr}\left(\operatorname{tr}_{\widehat{M}}\left(\rho_{P}(t) \rho_{\widehat{M}}\left(t_{0}\right)\right) \rho_{\breve{M}}\left(t_{0}\right)\right)}
$$

Entanglement of the Quantum system is:

$$
E_{R E}:=\min \left\{E_{R E}\left(\rho_{N}\right)\right\}_{N \in \mathcal{T}}
$$

Where

$$
E_{R E}\left(\rho_{N}\right):=\min \left\{S\left(\rho_{N} \|\left(\prod_{\alpha_{i} \in \Lambda} \operatorname{dim} \mathbb{H}_{\alpha_{i}}\right) \rho_{\widehat{N}} \rho_{\widetilde{N}}\right)\right\}_{\substack{\widehat{N} \cup \breve{N}=N \\, \widehat{N}, \mathbb{N} \neq N \\ \widehat{N}, \widehat{N} \in T}}
$$

And $S(* \| *)$ is the relative entropy. Considering the distance function, $\mathcal{D}$ as $S$ and now making the following observation.For each pair of $M, P \in \mathcal{T}$ we can find a $N \in \mathcal{T}$ such that $\rho_{N}:=\varrho^{x}\left(\rho_{P} \mid \rho_{M}\left(t_{0}\right)\right)$ and for each pair of $\widehat{M}, \breve{M}$ we can find a pair of $\widehat{N}, \widehat{N}$ satisfying all the above conditions and $\rho_{\widehat{N}} \rho_{\widetilde{N}}:=\varrho^{x}\left(\varrho^{x}\left(\rho_{P} \mid \rho_{\widehat{M}}\left(t_{0}\right)\right) \mid \rho_{\breve{M}}\left(t_{0}\right)\right)$, due to way more constrains in the definition of QMICE the converse is not true that's why

$$
E_{R E}\left(\varrho^{x}\left(\rho_{P} \mid \rho_{M}\left(t_{0}\right)\right)\right) \leq \varphi\left(P \mid M_{s}\right)
$$

Hence

$$
E_{R E} \leq \varphi^{\max }\left(\rho_{M}\left(t_{0}\right)\right)
$$

Hence entanglement generate concept. 
We define Entanglement information, $\mathcal{E} \mathcal{J}$ by replacing QMICE with $E_{R E}$ in $\mathcal{Q} \mathcal{C J}$ and it is not difficult to see that $\mathcal{E} \mathcal{J} \leq \mathcal{Q} \mathcal{C}$ J. Similarly, with the definition that we have of $\Delta$,

$$
\Delta(\mathcal{Q} \mathcal{S}(\mathcal{U}), \mathcal{Q} \mathcal{S}(\mathcal{V})):=|\mathcal{Q} \mathcal{J}(\mathcal{C S}(\mathcal{U}))-\mathcal{Q} \mathcal{J}(\mathcal{C S}(\mathcal{V}))|
$$

We define $\Phi_{\mathcal{E}}(\mathcal{U})$ for the subsystem $\mathcal{U}$ by replacing $\mathcal{Q} \mathcal{C J}$ with $\mathcal{E} \mathcal{J}$ in the definition of $\Phi_{Q}(\mathcal{U})$. And we get $\Phi_{\mathcal{E}}(\mathcal{U}) \leq \Phi_{\mathcal{Q}}(\mathcal{U})$ for all $\mathcal{U} \cdot \max \left\{\Phi_{\mathcal{E}}(\mathcal{U})\right\}_{\mathcal{U} \subset \mathcal{T}} \leq \max \left\{\Phi_{\mathcal{Q}}(\mathcal{U})\right\}_{\mathcal{U} \in \mathcal{T}}$. Which implies $\Phi_{\mathcal{E}}^{\max }(\mathcal{T}) \leq \Phi_{Q}^{\max }(\mathcal{T})$, where $\Phi_{\mathcal{E}}^{\max }(\mathcal{T}):=\max \left\{\Phi_{\mathcal{E}}(\mathcal{U})\right\}_{\mathcal{U} \in \mathcal{T}}$. Which means if entanglement occurs it creates consciousness, which would explain the breakdown of wavefunction due to it.

\section{Discussion and Conclusion}

For a set of elements $\Lambda$ (e.g. degrees freedom), its open subset is a mechanism, $M$ (in case of discrete topology all the subsets are open) and set of open sets i.e. topology, $\mathcal{T}$ is system. The QIIT is the functor, $\mathcal{F}$ from the topological category i.e. category whose set of object is the topology $\mathcal{T}$ to the linear category, i.e. the category whose objects are the set Hilbert spaces. The state of endomorphism between on the Hilbert spaces. Cause- effect repertoire of a given mechanism, $M$ is the its conational density matrix, $\varrho^{x}\left(\rho_{P} \mid \rho_{M}\left(t_{0}\right)\right)$ of other mechanisms, $P$ knowing a given mechanism, $M$ in a given particular state. Given a distance function on density matrix space, $\mathcal{D}_{Q}$. The minimum distance between the repertoire of a mechanism, $\varrho^{x}\left(\rho_{P} \mid \rho_{M}\left(t_{0}\right)\right)$ and repertoire of its sub-mechanism and then considering that repertoire as perview for calculating the repertoire for its complement sub-mechanism, $\varrho^{x}\left(\varrho^{x}\left(\rho_{P} \mid \rho_{\widehat{M}}\left(t_{0}\right)\right) \mid \rho_{\widetilde{M}}\left(t_{0}\right)\right)$. The maximum of all quantum cause effect integrated information in QMICE and if it is non zero then it creates concept. The concept is 3-tuple of QMICE, respective cause repertoire and effect repertoire. Conceptual structure is a subsystem (subset of topology) is tuple over all states and mechanisms over that system. Conceptual information is measure of information in conceptual structure and increases with increase in QMICE of concept of conceptual structure and with increase in number of concepts of conceptual structure and could be defined as:

$$
\mathcal{Q} \mathcal{J}(\mathcal{C S}(\mathcal{U})):=\sum_{\substack{M \in U \\ t_{0} \in R}} \varphi^{\max }\left(\rho_{M}\left(t_{0}\right)\right) \mathcal{Q} \mathcal{J}(\mathcal{U})
$$

Where $\mathcal{Q J}(\mathcal{U}):=\max \left\{Q \operatorname{ce}\left(\rho_{P} \mid \rho_{M}\left(t_{0}\right)\right)\right\}_{\substack{P \in T \\ M \in U}}$. Quantum Integrated conceptual information is the minimum of the distance between conceptual structure of a sub-system, $\mathcal{U}$ and the subtotal of the conceptual structure of its constituents, $\widehat{u}$, $\breve{u}$ and is synonymous to Quantum integrated cause-effect information and can be defined as,

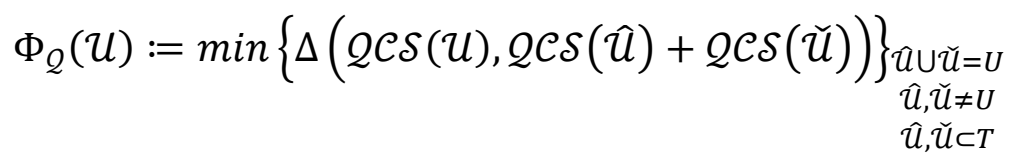

Where $\Delta$ is distance function over space of conceptual structures and can be best defined as, 


$$
\Delta(\mathcal{Q} \mathcal{S}(\mathcal{U}), \mathcal{Q} \mathcal{S}(\mathcal{V})):=|\mathcal{Q} \mathcal{J}(\mathcal{C S}(\mathcal{U}))-\mathcal{Q} \mathcal{J}(\mathcal{C S}(\mathcal{V}))|
$$

Where $\mathcal{U}, \mathcal{v} \in \mathcal{T}$. Quantum Maximum of integrated conceptual information is measure of consciousness.

\section{Declarations}

\section{Ethics approval and consent to participate}

Not applicable

\section{Consent for publication}

I give my consent to publication

\section{Availability of supporting data}

Not applicable. If your manuscript does not contain any data, please state 'Not applicable' in this section.

\section{Competing interests}

No competing interests

\section{Funding}

No funding

6. Authors' contributions

I solely contributed in every capacity

\section{Acknowledgements}

Not applicable

\section{Reference}

1. Sharma, S. (2021, January 1). Classical Mathematical Integrated Information Theory. https://doi.org/10.31219/osf.io/qf58g

2. Oizumi, M., Albantakis, L., \& Tononi, G. (2014). From the Phenomenology to the Mechanisms of Consciousness: Integrated Information Theory 3.0. PLoS Computational Biology, 10(5). https://doi.org/10.1371/journal.pcbi.1003588

3. Griffiths, R. (2001). Consistent Quantum Theory. Cambridge: Cambridge University Press. doi:10.1017/CBO9780511606052

4. Kleiner, J. Mathematical Models of Consciousness. Entropy 2020, 22, 609. 
5. Bengtsson, I., \& Zyczkowski, K. (2006). Geometry of Quantum States: An Introduction to Quantum Entanglement. Cambridge: Cambridge University Press. doi:10.1017/CBO9780511535048

6. G. Tononi, BMC Neuroscience 5, 42 (2004).

7. M. Oizumi, L. Albantakis, and G. Tononi, PLOS Computational Biology 10, 5 (2014)

8. K. Kremnizer and A. Ranchin, Found. Phys. 45, 889 (2015).

9. L. Albantakis and G. Tononi, Entropy 17, 5472 (2015).

10. M. Tegmark, Chaos, Solitons \& Fractals 76, 238 (2015).

11. M. Tegmark, PLOS Computational Biology 12, 11 (2016).

12. G. Tononi and C. Koch, Phil. Trans. R. Soc. B 370, 20140167 (2015)

13. E. Lieb, D. Robinson, Commun. Math. Phys. 28, 251 (1972)

14. Sharma, S. (2020, December 18). Entropic Integrated Information Theory-Theory of Consciousness. https://doi.org/10.31219/osf.io/uswmr

15. Munkres, J. R. (2000). Topology. Upper Saddle River, NJ: Prentice Hall, Inc.

16. https://doi.org/10.1007/978-1-4757-1793-8_1

17. Tononi, G. An information integration theory of consciousness. BMC Neurosci 5, 42 (2004). https://doi.org/10.1186/1471-2202-5-42

18. Science Translational Medicine 14 Aug 2013:Vol. 5, Issue 198, pp. 198ra105 DOI: 10.1126/scitranslmed.3006294

19. Petz D. (2008) Prerequisites from Quantum Mechanics. In: Quantum Information Theory and Quantum Statistics. Theoretical and Mathematical Physics. Springer, Berlin, Heidelberg. https://doi.org/10.1007/978-3-540-74636-2_25.

20. Petz D. (2008) Information and its Measures. In: Quantum Information Theory and Quantum Statistics. Theoretical and Mathematical Physics. Springer, Berlin, Heidelberg. https://doi.org/10.1007/978-3-540-74636-2_36.

21. \#Petz D. (2008) Entanglement. In: Quantum Information Theory and Quantum Statistics. Theoretical and Mathematical Physics. Springer, Berlin, Heidelberg. https://doi.org/10.1007/978-3-540-74636-2_47. 